\title{
Caries-risk profiles in Italian adults using computer caries assessment system and ICDAS
}

\section{Giovanna CARTA(a) \\ Maria Grazia CAGETTI(b) \\ Fabio $\mathrm{COCCO}^{(\text {b) }}$ \\ Silvana SALE(a) \\ Peter LINGSTRÖM(c) \\ Guglielmo CAMPUS(b)}

(a) University of Sassari, Department of Surgery, Microsurgery and Medicine Sciences, Sassari, Italy.

(b) University of Milan, Department of Health Science, WHO Collaborating Centre for Epidemiology and Community Dentistry, Milan, Italy.

(c) University of Gothenburg, Department of Cariology, Institute of Odontology, The Sahlgrenska Academy, Gothenburg, Sweden.

Declaration of Interests: The authors certify that they have no commercial or associative interest that represents a conflict of interest in connection with the manuscript.

Corresponding Author:

Guglielmo Campus

E-mail:gcampus@uniss.it

DOI: 10.1590/1807-3107BOR-2015.vol29.0126

Submitted: Jan 08, 2015

Accepted for publication: Jun 23, 2015

Last revision: Aug 31, 2015

\begin{abstract}
The aim of this study was to examine the correlation among socio-behavioral factors, caries status and caries risk, calculated through Cariogram, in an adult population. Four hundred eighty subjects (mean age 40.73, SE \pm 0.33 ) randomly selected from the municipal electoral registry consented to participate in the survey. Subjects were examined, and the International Caries Detection Assessment System (ICDAS) index was registered. A highly structured questionnaire was submitted to investigate (1) personal data (i.e., age, gender, educational level, job categorization), (2) life-style behavior (i.e., smoking and dietary habits), and (3) oral health behavior (i.e., tooth brushing, use of fluoride and dental check-up frequency). An evaluation of the mutans streptococci concentration in saliva was also performed. Information on caries-related factors was entered into the Cariogram in order to generate an individual caries risk profile for each subject. Multinomial logistic regression was performed using Cariogram levels as the dependent variable. The possible correlated variables were analyzed using the principal component analysis (PCA). Considering ICDAS scores, $5.62 \%$ of the sample had at least an initial decay (ICDAS $=1-2$ ), whereas $40.83 \%$ of the sample presented at least one moderate decay $($ ICDAS $=3-4$ ) and $17.08 \%$ a severe decay (ICDAS $=5-6$ ). Decay at ICDAS levels 5-6 and more than 5 missing teeth were statistically associated with Cariogram scores $(\mathrm{OR}=2.36,95 \% \mathrm{CI}=1.83-3.03$ and $\mathrm{OR}=1.43$, $95 \% \mathrm{CI}=1.13-1.82$, respectively). The results suggest that the Cariogram model was able to identify caries-related factors in an adult population. A direct association among the risk categories from Cariogram, the caries status and some socio-behavioral variables was verified.
\end{abstract}

Keywords: Dental Caries; Epidemiology; Adult.

\section{Introduction}

Although children are the primary recipients of caries prevention programs in the Western world, dental caries is still one of the primary causes of tooth loss among adults. ${ }^{1,2,3}$ Among the clinical risk factors, mutans streptococci play an important role in the development of dental caries. However, the focus on the non-biological determinants of the disease (socio-behavioral and environmental factors) is essential. ${ }^{5} \mathrm{~A}$ lower educational level has been statistically associated with greater severity of dental caries in adults. ${ }^{6,7}$ Among the prediction models available, ${ }^{8}$ Cariogram 
software has been developed and validated to illustrate graphically the chance of an individual avoiding caries in the near future. ${ }^{9,10,11,12}$ Cariogram has been used to predict caries in children, ${ }^{12}$ adults ${ }^{13,14,15,16,17,18,19}$ and the elderly, ${ }^{20}$ and the scientific evidence regarding its prediction efficiency is still questionable. For adults, despite some studies reporting a high predictive value for Cariogram risk levels, ${ }^{13,18}$ other studies have not found a correlation between caries experience and any single risk factor proposed by Cariogram. ${ }^{17,19}$ The use of Cariogram in an adult population might be useful to try to modify caries-related behaviors and increase patients' motivation.

Common outcomes used in studying the epidemiology of dental caries, such as DMFT and DMFS indices, may be insufficient for investigating the risk factors that lead to specific patterns of decay. ${ }^{21,22}$ The International Caries Detection Assessment System (ICDAS), based on the best available evidence for detecting early and later stage caries severity, should lead to the acquisition of better quality information to support decision-making at both individual and public health levels. ${ }^{22,23,24}$ For clinical application, the ICDAS Foundation developed an ICDAS code with merged categories called the International Caries Classification and Management System (ICCMS $\left.{ }^{\mathrm{TM}}\right){ }^{25}$

The aim of this study was to examine the correlation among socio-behavioral factors, caries status and caries risk, calculated through Cariogram, in an adult population.

\section{Methodology Study design}

The present study was carried out in Sassari (Sardinia, Italy) and lasted 14 months, from September 2012 to October 2013. The study design was registered (2013_01_21_a) by the Sassari local office of the National Bioethics Committee.

Data from the Italian National Institute for Statistics for 2011 showed the number of 35-45 year-olds in the town of Sassari to be 22,614. A power analysis was performed using $G^{*}$ Power 3.1.3 for Apple, logistic regression, an odds ratio of 1.5 , and an error probability of 0.04 , and the total sample size was set at 432 . A letter explaining the purpose of the study and the informed consent were distributed to $5 \%$ (1131 subjects) of the age group considered living in Sassari through the collaboration of the municipal electoral registry office; 577 subjects ( $51.02 \%$ acceptance rate) agreed to participate, 82 did not attend the examination and 15 did not completely fill out the questionnaire (Figure 1).

\section{Questionnaire}

A simplified structured self-compiled questionnaire and an informed consent form were submitted to the participants. The questionnaire was previously used in a large sample (2908 subjects) of Italian soldiers. ${ }^{4}$ It was divided into different domains: (1) personal data (i.e., age, gender, educational level, job categorization), (2) life-style behavior (i.e., smoking and dietary habits), and (3) oral health behavior (i.e., tooth brushing, use of fluoride, and frequency of dental check-ups).

\section{Caries assessment}

One calibrated examiner $(\mathrm{GiC})$ performed all the dental screenings. Intra- and inter-examiner reliabilities were assessed before the beginning of the survey by examining and re-examining (after 72 h) 55 study participants. Inter-examiner reliability was evaluated using fixed-effects analysis of variance in comparison with benchmark values (GC). Intra-examiner reliability was assessed as

Population object of the survey

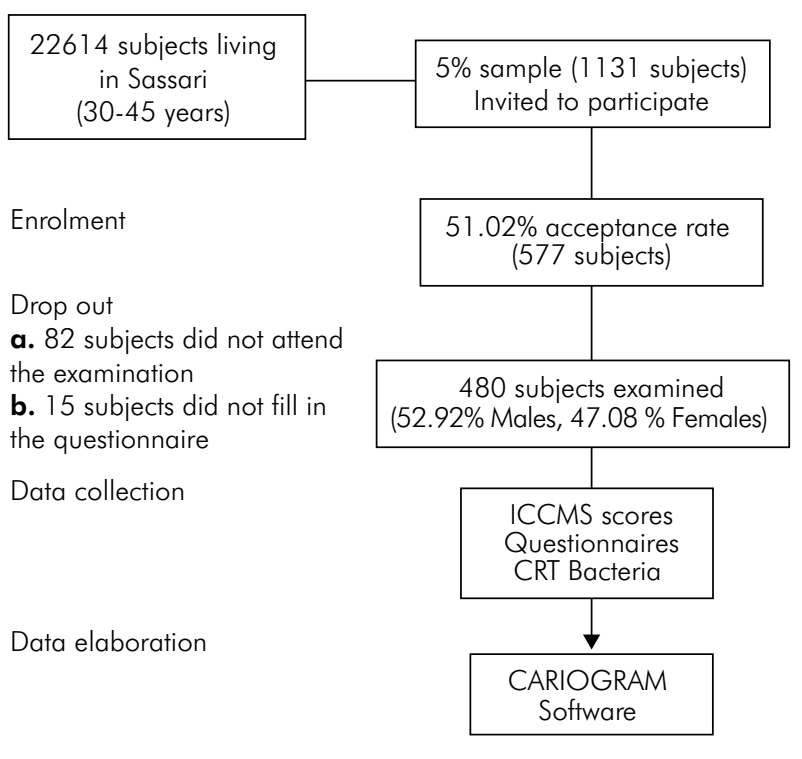

Figure 1. Flow chart of the survey. 
the percentage of agreement using Cohen's kappa statistic. ${ }^{26}$ Good inter-examiner reliability was found for the ICCMS ${ }^{\mathrm{TM}}$ (merged ICDAS) score 1-2, 3-4 and $5-6$, with no significant difference from benchmark values $(p=0.15)$ and a low mean square of error $(0.47)$. Intra-examiner reliability was also high (Cohen's Kappa statistic $=0.88)$. Subjects were examined sitting on an ordinary chair, with a mouth mirror and a Community Periodontal Index probe under optimal lighting. Subjects were categorized according to maximum ICDAS scores.

The number of missing teeth due to caries was categorized as follows: no missing teeth, 1-2 missing teeth, 3-5 missing teeth and more than 5 missing teeth. The presence of fillings was classified as: no fillings, mainly tooth-colored restorations, mainly amalgam restorations and the presence at least of one crown. Plaque index was assessed in the buccal surface using the Silness \& Löe's scale.

\section{Microbiological evaluation}

After the clinical examination, an evaluation of the mutans streptococci (MS) concentration in saliva was performed. No-stimulated whole saliva was collected over 150 seconds in sterile vials (Nunc, Kamstrup, Denmark). The samples were processed within 45 minutes after collection, and the analysis was carried out using CRT BACTERIA (Ivoclar, Vivadent AG, Schaan, Liechtenstein). The presence of mutans streptococci over $10^{5} \mathrm{CFU} / \mathrm{ml}$ suggests a high caries risk.

\section{Caries risk assessment using Cariogram}

Information on caries-related factors was collected and entered into Cariogram. ${ }^{9,10,11}$ The six caries-related factors listed below were included in the Cariogram software. ${ }^{9,10,11}$

Caries experience was recorded using the WHO basic report enclosed in the ICDAS evaluation, which allowed the calculation of the DMFT of the population.

1. Diet content (from questionnaires);

2. Diet frequency (from questionnaires);

3. Salivary levels of mutans streptococci (from CRT Bacteria);

4. Plaque amount and grade of oral hygiene (from clinical examination);
5. Related general diseases (from questionnaires);

6. Fluoridation program (from questionnaires).

A score of 1 in all cases was assigned for clinical judgment in order to produce a standard value.

The "chance to avoid new caries in the future" was calculated, and the subjects were divided into three groups: low risk (61-80\%), medium risk (41-60\%) and high risk (21-40\%).

\section{Statistical analyses}

Descriptive statistics and crosstabs were calculated to investigate the relationship between dental health and different risk factors. The reference for each variable was set as follows: gender (male); caries status (ICDAS 5-6); type of dental treatment (presence of at least one crown); number of missing teeth due to caries (more than five teeth); educational level (middle school degree); occupational status (unemployed); smoking habits (yes); frequency of toothbrushing (once a day); and frequency of dental check-ups (when in pain).

A chi-square test was conducted for each variable and the level of caries risk measured by Cariogram. Multinomial logistic regression was performed using Cariogram levels as the dependent variable. The possible correlated variables were analyzed using the PCA. ${ }^{27}$ The performance of Cariogram in predicting caries in the near future was evaluated by receiver operating characteristic (ROC) analysis. ${ }^{28}$ The p-values $<0.05$ were considered statistically significant. All data were analyzed using v10.1 software for Macintosh produced by STATA ${ }^{\circledR}$ (STATA Corporation, College Station, Texas).

\section{Results}

The study population consisted of 480 subjects (mean age $40.7, \mathrm{SE} \pm 0.3$ ), $52.9 \%$ males and $47.1 \%$ females The caries prevalence in the sample was $57.9 \%$. The sample distribution by gender across the clinical data for ICDAS scores (initial caries ICDAS 1-2, moderate caries ICDAS 3-4, and extensive caries ICDAS 5-6) and dental treatment is displayed in Table 1. Almost half of the sample $(47.9 \%)$ had at least one missing tooth due to caries. The mean number of sound teeth registered was 24.8 (SE \pm 0.2$)$. Each subject presented an average of $3.7(\mathrm{SE} \pm 0.1)$ tooth-colored restorations, 
3.0 (SE \pm 0.1$)$ amalgam restorations and 1.58 (SE \pm 0.14 ) crowns. Regarding the questionnaires, $17.9 \%$ of subjects had graduated from middle school, $47.3 \%$ (most) had graduated from high school, and 34.8\% (a quite high percentage) had a university degree. Additionally, $60.4 \%$ of the sample brushed their teeth three times a day. Only $26.7 \%$ of the subjects were smokers, and most of them had been smoking for more than 5 years (data not in table).

Relating to caries risk, the majority of the sample (78.1\%) presented a medium caries risk $(41-60 \%$ of chance to avoid new caries lesions in the future), $18.8 \%$ had a high caries risk ( $<41 \%$ of chance to avoid new caries lesions in the future) and $3.1 \%$ had a low caries risk $>60 \%$ of chance to avoid new caries lesions in the future). Table 2 shows the results of a chi-square test between the level of caries risk, calculated through Cariogram software, and background variables. A statistically significant association was evident between the caries risk and ICDAS score $(p<0.01)$. A statistically significant association was found between the level of caries risk and the following factors: number of missing teeth due to caries $(p=0.01)$, educational level $(p=0.04)$, smoking habit $(p=0.01)$, frequency of toothbrushing $(\mathrm{p}=0.02)$ and frequency of dental check-ups $(p<0.01)$.

A multinomial logistic regression model was run for caries risk factors (Table 3), and the Cariogram score was used as a dependent variable. The presence of caries lesions at a ICDAS level 5-6 and the presence of more than 5 missing teeth were statistically associated with Cariogram scores (OR $=2.36,95 \% \mathrm{CI}=1.83-3.03$ and $\mathrm{OR}=1.43,95 \% \mathrm{CI}=1.13-1.82$, respectively).
PCA was performed on the data set, first on the total sample and in the two main groups stratified by Cariogram scores (caries medium-risk group and high-risk group); the low caries risk group was too small, and PCA was not performed. In all PCAs, the first two eigenvalues, obtained from the distance matrix between groups, collectively accounted for more than $66.0 \%$ of the total variance $(86.1 \%$; 59.4 and $26.8 \%$, respectively in the total sample; $52.5 \%$ and $21.1 \%$ respectively and $66.3 \%$ in the high-risk group $42.7 \%$ and $23.6 \%$ two eigenvalues respectively). Figure 2 displays the Orthogonal Rotation (Varimax) of the first two principal coordinates in the total sample and in the two main groups stratified by Cariogram scores. In the total sample, the Cariogram score, the Bacteria and the Susceptibility sectors from Cariogram tended to form a separate cluster with a high goodness of fit (63.7); also, number of missing teeth due to caries, smoking habit and circumstances from Cariogram tended to form another cluster with a high goodness of fit (61.8); however, level of education, gender and job categorization were clearly separated from the other variables. Severe caries status (ICDAS 5-6), number of missing teeth due to caries higher than 5, unemployment, smoking and male gender tended to form a cluster with a medium-high goodness of fit (0.5-0.7) in the high-risk group. In the medium-risk group, no clear cluster was identified, and all variables stood separated from each other. The sensitivity and specificity between ICDAS scores and Cariogram, measured by ROC analysis, were 0.8 and 0.9 , respectively, so the gain in certainty was 1.7 , while the area under the ROC curve was 0.9 (data not in table).

Table 1. Sample distribution of clinical data by gender.

\begin{tabular}{lccc}
\hline & Males percentage & Females percentage & Total sample percentage \\
\hline Caries Prevalence & 64.43 & 62.12 & 63.54 \\
Initial lesion (ICDAS1-2) & 6.05 & 5.82 & 5.62 \\
Moderate lesion (ICDAS 3-4) & 40.32 & 43.54 & 40.83 \\
Extensive lesion (ICDAS 5-6) & 17.54 & 16.14 & 17.08 \\
Dental treatment & 4.49 & 4.19 & 4.39 \\
One tooth-colored restoration & 33.28 & 33.44 & 33.31 \\
One amalgam restoration & 28.64 & 30.36 & 29.58 \\
One missing tooth due to caries & 48.01 & 49.91 & 47.92 \\
One crown & 33.16 & 32.26 & 32.72 \\
\hline
\end{tabular}


Table 2. Sample distribution by caries risk level (low, medium or high) according to gender, caries status, and treatment educational level, occupational status and behavioural habit.

\begin{tabular}{|c|c|c|c|}
\hline & $\begin{array}{c}\text { Low Caries Risk } \\
\mathrm{n}(\%)\end{array}$ & $\begin{array}{c}\text { Medium Caries Risk } \\
\mathrm{n}(\%)\end{array}$ & $\begin{array}{c}\text { High Caries Risk } \\
\mathrm{n}(\%)\end{array}$ \\
\hline \multicolumn{4}{|l|}{ Gender } \\
\hline Males & $8(1.66)$ & $192(40.00)$ & $54(11.25)$ \\
\hline Females & $7(1.46)$ & $183(38.12)$ & $36(7.50)$ \\
\hline \multicolumn{4}{|l|}{$\chi_{(2)}^{2}=2.26, p=0.32$} \\
\hline \multicolumn{4}{|l|}{ Caries status } \\
\hline Caries-free (ICDAS 0) & $11(2.29)$ & $156(32.50)$ & $8(1.66)$ \\
\hline Initial caries (ICDAS 1-2) & $1(0.21)$ & $24(5.00)$ & $2(0.42)$ \\
\hline Moderate caries (ICDAS 3-4) & $3(0.62)$ & $148(30.83)$ & $45(9.37)$ \\
\hline Severe caries (ICDAS 5-6) & $0(0.00)$ & $47(9.79)$ & $35(7.29)$ \\
\hline \multicolumn{4}{|l|}{$\chi_{(6)}^{2}=65.13, p<0.01$} \\
\hline \multicolumn{4}{|l|}{ Type of dental treatments } \\
\hline No-treatments & $0(0.00)$ & $18(3.75)$ & $3(0.62)$ \\
\hline Tooth-colored restorations & $3(0.62)$ & $126(26.25)$ & $31(64.58)$ \\
\hline Amalgam restorations & $6(1.25)$ & $112(23.33)$ & $24(5.00)$ \\
\hline Crowns & $6(1.25)$ & $119(24.79)$ & $32(8.00)$ \\
\hline \multicolumn{4}{|l|}{$\chi_{(6)}^{2}=3.27, p=0.77$} \\
\hline \multicolumn{4}{|c|}{ Number of missing teeth due to caries } \\
\hline No-missing teeth & $13(2.71)$ & $201(41.87)$ & $36(7.50)$ \\
\hline $1-2$ missing teeth & $2(0.42)$ & 95 (19.79) & $18(3.75)$ \\
\hline 3-5 missing teeth & $0(0.00)$ & $54(11.25)$ & $26(5.42)$ \\
\hline$>5$ missing teeth & $0(0.00)$ & $25(5.21$ & $10(2.08)$ \\
\hline \multicolumn{4}{|l|}{$\chi_{(6)}^{2}=22.54, p=0.01$} \\
\hline \multicolumn{4}{|l|}{ Educational level } \\
\hline Middle school degree & $0(0.00)$ & $67(13.96)$ & $19(3.96)$ \\
\hline High school degree & $5(1.04)$ & $185(38.54)$ & $37(7.71)$ \\
\hline University degree & $10(2.08)$ & $123(25.62)$ & $34(7.08)$ \\
\hline \multicolumn{4}{|l|}{$\chi_{(4)}^{2}=9.91, p=0.04$} \\
\hline \multicolumn{4}{|l|}{ Occupational status } \\
\hline Unemployed & $1(0.21)$ & 59 (12.29) & $18(3.75)$ \\
\hline Housewife & $6(1.25)$ & 85 (17.71) & $25(5.21)$ \\
\hline Technician/clerk & $4(0.83)$ & $168(35.00)$ & 35 (7.29) \\
\hline Professional & $4(0.83)$ & $63(13.12)$ & $12(2.50)$ \\
\hline \multicolumn{4}{|l|}{$\chi^{2}(6)=7.15, p=0.31$} \\
\hline \multicolumn{4}{|l|}{ Smoking habits } \\
\hline No-smokers & $15(3.12)$ & $280(58.33)$ & 57 (1 1.87) \\
\hline Smokers & $0(0.00)$ & 95 (19.79) & $33(6.87)$ \\
\hline \multicolumn{4}{|l|}{$\chi_{(2)}^{2}=10.40, p=0.01$} \\
\hline \multicolumn{4}{|l|}{ Frequency of toothbrushing } \\
\hline Once a day & $0(0.00)$ & $5(1.25)$ & $0(0.00)$ \\
\hline Twice a day & $2(0.41)$ & $133(27.71)$ & $48(10.00)$ \\
\hline 3 times a day & $13(2.71)$ & $236(49.16)$ & $41(8.54)$ \\
\hline 4 times a day & $0(0.00)$ & $1(0.21)$ & $0(0.00)$ \\
\hline \multicolumn{4}{|l|}{$\chi_{(6)}^{2}=14.47, p=0.02$} \\
\hline \multicolumn{4}{|l|}{ Frequency of dental check-ups } \\
\hline Each 6 month & $5(1.25)$ & $46(8.54)$ & $4(0.83)$ \\
\hline Once a year & $7(1.46)$ & $132(27.50)$ & $24(5.00)$ \\
\hline Once 2 years & $0(0.00)$ & $101(21.04)$ & $23(4.79)$ \\
\hline At pain & $3(0.62)$ & $96(20.00)$ & $39(8.12)$ \\
\hline$\chi_{(6)}^{2}=25.04, p<0.01$ & & & \\
\hline
\end{tabular}


Table 3. Multinomial logistic regression estimates for significantly associated caries risk covariates to Cariogram scores.

\begin{tabular}{lccc}
\hline Covariates & OR (SE) & p-value & $95 \% \mathrm{Cl}$ \\
\hline Caries status (ICDAS 5-6) & $2.36(0.30)$ & $<0.01$ & $1.83-3.03$ \\
Number of missing teeth (> 5 missing teeth) & $1.43(0.17)$ & $<0.01$ & $1.13-1.82$ \\
Smoking habits (Yes) & $1.28(0.16)$ & 0.36 & $0.76-2.14$ \\
Dental check-ups (At pain) & $1.28(0.16)$ & 0.04 & $1.00-1.65$ \\
Tooth brushing frequency (3 times a day) & $0.64(0.15)$ & 0.06 & $0.40-1.01$ \\
\hline
\end{tabular}

Number of observations: 480; Log likelihood: $-248.33, p<0.01$.

All cariogram scores ( $\mathrm{N}=480$ subjects)

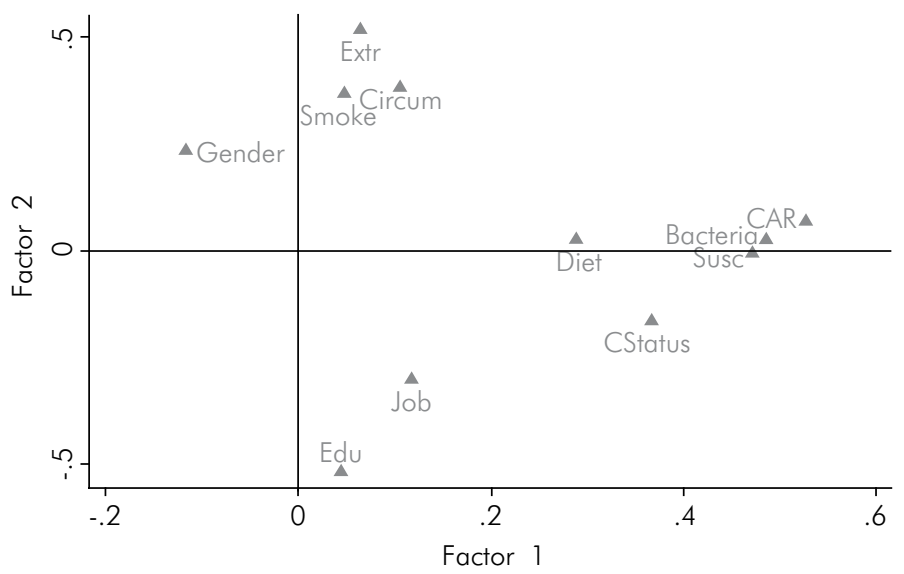

Factor 1: Coef.(SE) $2.94(0.19) p<0.01$

Factor 2: Coef.(SE) $1.38(0.09) \mathrm{p}<0.01$

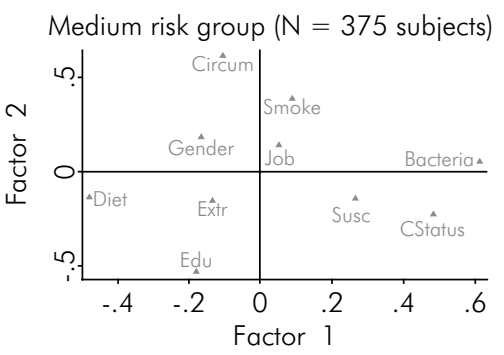

Factor 1: Coef.(SE) $1.92(0.14) p<0.01$

Factor 2: Coef.(SE) $1.23(0.09) p<0.01$

High risk group ( $\mathrm{N}=90$ subjects)

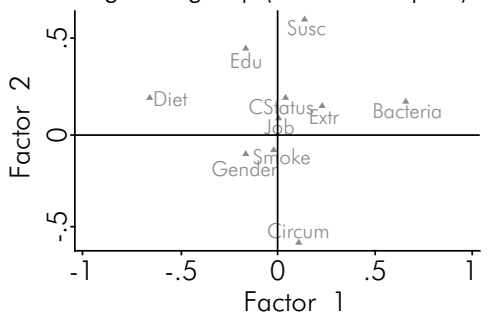

Factor 1: Coef.(SE) $2.06(0.31) \mathrm{p}<0.01$

Factor 2: Coef.(SE) $1.50(0.23) \mathrm{p}<0.01$

Extr: $>5$ extracted teeth due to caries; Gender: male; Smoke: smoking habits; Circum: circumstances in Cariogram analysis; Diet: diet frequency and Diet content; Job: occupational status (unemployed); Edu: level of education (middle school degree); Bacteria: salivary mutans streptococci; Susc: suscepEbility in Cariogram analysis; CAR: level of caries risk calculated through Cariogram; CStatus: caries status using ICDAS.

Figure 2. Principal Component Analysis. Orthogonal rotation (varimax).

\section{Discussion}

Because caries distribution is remarkably uneven, an accurate assessment of caries risk would allow a better use of limited resources. ${ }^{8}$ A computer caries assessment system might represent a helpful tool to assess caries risk before the development of the disease. ${ }^{910,11}$ A simplified computer caries assessment system, with the exclusion of two risk factors, may be used in clinical practice, when a full inclusion of risk factors is not achievable. ${ }^{13}$ Little information exists on the performance of Cariogram in adult individuals, $10,13,14,15,16,17,1,19$ so the aim of this study was to describe the relation among caries status, background variables and caries risk as calculated with Cariogram. More than two-thirds of the sample showed a medium risk, and most of the remaining sample showed a high risk of future caries development. These findings showed a statically significant association with the caries status and the number of missing teeth due to caries, measured using ICDAS criteria. Similar results were reported in Spanish young adults with a statistically significant association between the caries risk profile determined by Cariogram and past caries experience. ${ }^{16}$ Otherwise, in a high-caries adult population, different results were found, e.g., the Cariogram risk categories did not fit with caries 
experience. ${ }^{17}$ Such a result was probably due to the hypothesized poor ability of the Cariogram to properly clarify the association between caries risk and caries experience in a high-caries population. Nevertheless, a direct association between the categorized outcomes of the Cariogram and caries status was found in a group of Saudi adults with several dental restorations. ${ }^{14}$ Caries still remains an important reason for tooth loss, ${ }^{1,2,29}$ and the results of this paper noted the association between this variable and the caries risk profile measured by the Cariogram model. Moreover, the multinomial logistic regression outcome underlines an association between the Cariogram score and the higher ICDAS score and also the higher number of missing teeth.

Among background variables, educational level, smoking habit, frequency of toothbrushing and frequency of dental check-ups showed a statistically significant association with Cariogram risk groups. In particular, the educational level is one of the most frequently used socioeconomic indicator: a lower educational level has been statistically associated with a greater prevalence of dental caries. ${ }^{6,7}$ The finding of a statistically significant association among the Cariogram score and socioeconomic and behavioral variables is quite new and interesting because it highlights that even if the Cariogram model includes proximal risk factors for caries almost exclusively, distal risk factors, such as smoking and educational level, are strongly correlated with the risk assessed through the Cariogram.

PCA supports the concept that caries risk is divisible into patterns attributable to distinct risk factors that work in accord. In the total sample, two different clusters might be noted: the first centered on a Cariogram score with two Cariogram sectors, Susceptibility (fluoride program, saliva secretion and buffer capacity) and Bacteria (plaque amount and MS), while the second is the association of a high number of missing teeth due to caries, smoking habit and circumstances (caries experience and related diseases), which further supports the involvement of environmental risk factors on the caries pattern. The interpretation of the PCA on the Cariogram high-risk group is that those with a severe caries status (ICDAS 5-6), with a high number of missing teeth due to caries, who are unemployed, smoke and are male tend to be strictly associated with a distinct disease pattern. Socioeconomic indicators often have a heavy impact on biological factors, influencing variables such as diet or oral hygiene habits. 5,7,29 Therefore, it is possible to suggest that those who have no job are more likely to have more caries, lose more teeth due to caries, and have no economic resources to receive dental treatment because, in Italy, public dental services for adults are very limited and dental care has to be paid directly by the patient.

This study benefited from several strengths, as a large sample and the surface-level caries assessment that made the different modeling of caries patterns possible. A limitation would be the non-generalizability of the results. The studied population belonged to a medium-low socio-economic group living in an area with a low mean income per capita. It is possible that, in a population with different socio-economic and behavioral variables, distinct risk factors may have an influence on caries disease.

\section{Conclusion}

In conclusion, the Cariogram model was able to identify caries-related factors in an adult population. A direct association among the risk categories from Cariogram, the caries status and some socio-behavioral variables was verified.

\section{Availability of supporting data}

The authors are ready to provide supporting data upon request.

\section{Acknowledgments}

The authors would like to thank everyone who helped carry out this study, including the personnel of the municipal electoral registry office of the town of Sassari. 


\section{References}

1. Fure S. Ten-year incidence of tooth loss and dental caries in elderly Swedish individuals. Caries Res. 2003;37(6):462-9. doi:10.1159/000073401

2. Broadbent JM, Thomson WM, Poulton R. Progression of dental caries and tooth loss between the third and fourth decades of life: a birth cohort study. Caries Res. 2006;40(6):459-65. doi:10.1159/000095643

3. Vollmer WM, Papas AS, Bader JD, Maupomé G, Gullion CM, Hollis JF et al.; PACS Collaborative Research Group. Design of the Prevention of Adult Caries Study (PACS): a randomized clinical trial assessing the effect of a chlorhexidine dental coating for the prevention of adult caries. BMC Oral Health. 2010;10(1):23. doi:10.1186/1472-6831-10-23

4. Senna A, Campus G, Gagliani M, Strohmenger L. Socio-economic influence on caries experience and CPITN values among a group of Italian call-up soldiers and cadets. Oral Health Prev Dent. 2005;3(1):39-46.

5. Holst D, Schuller AA, Aleksejuniené J, Eriksen HM. Caries in populations - a theoretical, causal approach. Eur J Oral Sci. 2001;109(3):143-8. doi:10.1034/j.1600-0722.2001.00022.x

6. Paulander J, Axelsson P, Lindhe J. Association between level of education and oral health status in 35-, 50-, 65and 75-year-olds. J Clin Periodontol. 2003;30(8):697-704. doi:10.1034/j.1600-051X.2003.00357.x

7. Costa SM, Martins CC, Bonfim M L, Zina LG, Paiva SM, Pordeus IA et al. A systematic review of socioeconomic indicators and dental caries in adults. Int J Environ Res Public Health. 2012;9(10):3540-74. doi:10.3390/ijerph9103540

8. Tellez M, Gomez J, Pretty I, Ellwood R, Ismail AI. Evidence on existing caries risk assessment systems: are they predictive of future caries? Community Dent Oral Epidemiol. 2013;41(1):67-78. doi:10.1111/cdoe.12003

9. Petersson GH. Assessing caries risk: using the Cariogram model. Swed Dent J Suppl. 2003;(158):1-65.

10. Bratthall D, Hänsel Petersson G. Cariogram - a multifactorial risk assessment model for a multifactorial disease. Community Dent Oral Epidemiol. 2005;33(4):256-64. doi:10.1111/j.1600-0528.2005.00233.x

11. Campus G, Cagetti MG, Sale S, Carta G, Lingström P. Cariogram validity in schoolchildren: a two-year follow-up study. Caries Res. 2012;46(1):16-22. doi:10.1159/000334932

12. Cabral RN, Hilgert LA, Faber J, LealSC. Caries risk assessment in schoolchildren: a form based on Cariogram software. J Appl Oral Sci. 2014;22(5):397-422. doi:10.1590/1678-775720130689

13. Lee JH, Son HH, Kim HY, Chang J. Caries risk profiles of Korean dental patients using simplified Cariogram models. Acta Odontol Scand. 2013;71(3-4):899-905. doi:10.3109/00016357.2012.734416

14. Sonbul H, Al-Otaibi M, Birkhed D. Risk profile of adults with severe dental restorations using the Cariogram model. Acta Odontol Scand. 2008;66(6):351-7. doi:10.1080/00016350802325853

15. Aranibar Quiroz EM, Alstad T, Campus G, Birkhed D, Lingström P. Relationship between plaque $\mathrm{pH}$ and different caries-associated variables in a group of adolescents with varying caries prevalence. Caries Res. 2014;48(2):147-53. doi:10.1159/000355614
16. Ruiz Miravet A. Montiel Company JM, Almerich Silla JM. Evaluation of caries risk in a young adult population. Med Oral Patol Oral Cir Bucal. 2007;12(5):E412-8.

17. Giacaman RA, Miranda Reyes P, Bravo León V. Caries risk assessment in Chilean adolescents and adults and its association with caries experience. Braz Oral Res. 2013;27(1):7-13. doi:10.1590/S1806-83242013000100002

18. Celik EU, Gokay N, Ates M. Efficiency of caries risk assessment in young adults using Cariogram. Eur J Dent. 2012;6(3):270-9.

19. Petersson GH, Twetman S. Caries risk assessment in young adults: a 3 year validation of the Cariogram model. BMC Oral Health. 2015;15(1):17. doi:10.1186/1472-6831-15-17

20. Alian AY, McNally ME, Fure S, Birkhed D. Assessment of caries risk in elderly patients using the cariogram model. J Can Dent Assoc. 2006;72(5):459-63.

21. Shaffer JR, Polk DE, Feingold E, Wang X, Cuenco KT, Weeks DE et al. Demographic, socioeconomic, and behavioral factors affecting patterns of tooth decay in the permanent dentition: principal components and factor analyses. Community Dent Oral Epidemiol. 2013;41(4):364-73. doi:10.1111/cdoe.12016

22. Iranzo-Cortés JE, Montiel-Company JM, Almerich-Silla JM. Caries diagnosis: agreement between WHO and ICDAS II criteria in epidemiological surveys. Community Dent Health. 2013;30(2):108-11. doi:10.1922/CDH_3006Silla04

23. Ismail AI, Sohn W, Tellez M, Willem JM, Betz J, Lepkowski J. Risk indicators for dental caries using the International Caries Detectionand AssessmentSystem(ICDAS). Community DentOral Epidemiol. 2008;36(1):55-68. doi:10.1111/j.1600-0528.2006.00369.x

24. Braga MM, Oliveira LB, Bonini GA, Bönecker M, Mendes FM. Feasibility of the International Caries Detection and Assessment System (ICDAS-II) in epidemiological surveys and comparability with standard World Health Organization criteria. Caries Res. 2009;43(4):245-9. doi:10.1159/000217855

25. Pitts NB, Ekstrand KR. International Caries Detection and Assessment System (ICDAS) and its International Caries Classification and Management System (ICCMS): methods for staging of the caries process and enabling dentists to manage caries. Community Dent Oral Epidemiol. 2013;41(1):e41-52. doi:10.1111/cdoe.12025

26. Castiglia P, Campus G, Solinas G, Maida C, Strohmenger L. Children's oral health in Italy: training and clinical calibration of examiners for the National Pathfinder about caries disease. Oral Health Prev Dent. 2007;5(4):255-61. doi:10.3290/j.ohpd.a12793

27. Van Ooyen A. Theoretical aspects of pattern analysis. A simple introduction to principal component and cluster analysis. In: Dijkshoorn L, Towner KJ, Struelens M (Eds.). New approaches for the generation and analysis of microbial fingerprints. Amsterdam: Elsevier; 2001. p. 31-45.

28. Koepsell TD, Connell FA. Measures of gain in certainty from a diagnostic test. Am J Epidemiol. 1985;121(5):744-53. doi:10.1093/aje/121.5.744

29. Beltran-Aguilar ED, Barker LK, Canto MT, Dye BA, Gooch $\mathrm{BF}$, Griffin SO et al. Surveillance for dental caries, dental sealants, tooth retention, edentulism, and enamel fluorosis: United States, 1988-1994 and 1999-2002. Surveillance Summaries. 2005;54:1-44. 